\title{
EFFECT OF ILLEGAL ON-STREET PARKING ON TRAVEL TIMES IN URBAN ENVIRONMENT
}

\author{
Morillo Carbonell, Carlos ${ }^{1}$
}

Transport Engineer, B:SM

PhD. Campos Cacheda, José Magín

Transport Professor, UPC

\begin{abstract}
The aim of this paper is to evaluate the effect of the on-street illegal parking on the commercial travel time of the vehicles in the area.

The effect of the illegal parking in the travel time of the vehicles in the zone is analyzed in an urban scenario in order to quantify the negative impact that illegal parking implies to this, by itself, congested areas.

To achieve the objective of the paper, a $3 \times 3$ street model has been designed and evaluated for different situations.
\end{abstract}

In this sense, based on a traffic microsimulation model a bunch of scenarios have been considered in function of parameters referred to intensity of vehicles, illegal on street parking level and location of the illegals.

Based on the scenarios mentioned, it has been analyzed the effect that the different parameters have on the commercial speed of the vehicles in order to have a first set of information that permits how to act to reduce the effect of illegal on street parking.

The results obtained in this article will be able to be used in next steps in order to define direct and indirect reduction strategies referred to illegal on street parking effect

\section{Highlights}

- Effect of illegal on-street parking in travel times

- The behavior of illegality depends on the location of the double parking

- Urban modeled theoretical schemes

\section{Keywords}

On-street parking, bottleneck, congestion, travel time, illegal parking

\footnotetext{
${ }^{1}$ Corresponding author: cmorillo@bsmsa.cat

Address: c/Valls I Taberner 14bis 7 2, 08006, Barcelona, Spain
} 


\section{Introduction}

The total amount of daily trips made in a town is outstanding due to urban areas are the most important poles of economic activity in a region and, therefore, where most of the trips end.

The trips made by private vehicles performed through the city represent around $30 \%$ and a $50 \%$ of all trips are done in the metropolitan area; however, important variations are registered depending on the city (for example, according to different sources, referred only to the trips made through the city, private vehicles represents almost $60 \%$ in Brussels but nearly $15 \%$ in Barcelona).

Regarding to this, all journeys, including private vehicles public transport and soft mode, are made, obviously, to achieve a concrete destination, usually predefined before the beginning of the trip.

In private vehicle journeys, it is worthy to remark that almost all trips carried out begin without a parking lot secured (only people who made a reservation or own/rent a car park lot have it). This problem only appears with private transport because public transport as well as soft modes requires no parking lot to finalize their journeys.

The lack of secured space to park in destination in most of the cases implies that the driver must search for an on street lot or off street lot when arrives to the end of the trip, commonly known as cruising for parking.

In the case of two-wheel vehicles, this lack of secured space does not represent, actually, an important problem to local administration because this kind of vehicle is allowed to park on the sidewalk - completely free (except some cases as Westminster - United Kingdom - where a fixed rate is required to park on sidewalk - permitting an offer considerably greater than demand.

Despite the problem related to the 2-wheel vehicles is not significant, in the case of 4wheel vehicles the problem appears due to they need a greater amount of space to park. This requirement implies that, often, the offer does not suit the demand due to multiple reasons such as lack of physical space, economic cost of a parking lot, location of the lot, need of cruising for parking, etc. implying the appearance of on-street illegal parking whether it is on the sidewalk or on the side of the road (Shoup, 2005).

The appearance of illegality implies the reduction of the commercial speed and an increase of the congestion making an inefficient use of the infrastructure (according to Willeke,1984).

The aim of this paper is to calculate the effect for the vehicles circulating in an urban environment when travel time effects related to illegality on parking appear.

In order to calculate the travel time effects, the key point is to evaluate the volume of illegal parking, the total amount of flow as well as the location of it. This calculation will allow to study strategic measures to reduce and mitigate the negative impact that local administrations can implement (as suggested by J.Wang, F.Chien, 2012 and A.Albiol et al., 2015)

For this reason, a wide simulation of a concrete urban case has been considered where different illegal scenarios (volume, flow and illegal location) have been considered. 


\section{Materials and methods}

In order to develop the current paper, a vehicle microsimulation software tool has been used in order to evaluate the different scenarios as well as the conditions related to them with the goal to obtain the final results.

In this sense, first of all, the different studio cases to simulate have been defined. A general orthogonal, according to Basic urban network in Barcelona, case has been chosen just to adapt it to a real continuous model. The following figure shows the case elaborated.

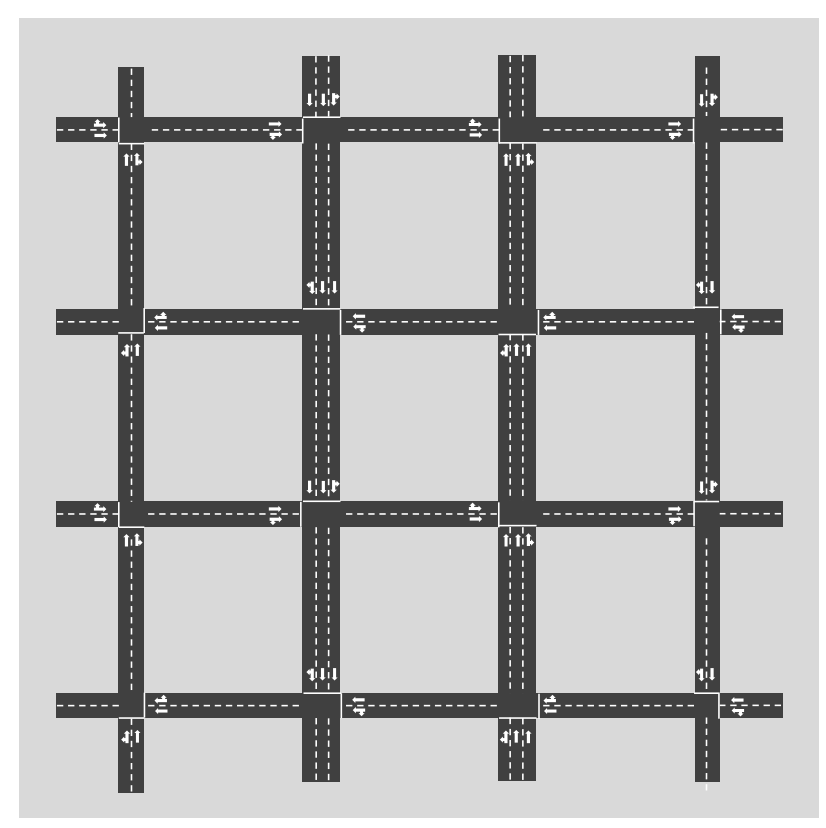

Figure 1. Urban model scheme used

Once the scenario has been defined, the boundary conditions have been defined. These conditions are the circulation flow referred to each lane as well as the origin and destinations of each of the vehicles.

Next to boundary conditions, it have been defined the specific scenario conditions like existing illegal parking and the location of it in the urban area.

\subsection{Case definition}

For all the scenarios chosen, the model considered is an orthogonal road distribution of $3 \times 3$ roads ( 9 neighborhoods) with one direction streets. There are 4 main roads located at the center of the scheme ( 2 with 3 lane road each and 2 more with 2 lanes) and the rest are considered secondary roads (all with 2 lanes).

All the intersections are controlled by traffic lights. The main roads have the highest level of priority in the model. 


\subsection{Definition of boundary conditions}

In order to fulfil the goal to analyze the effects of illegal on-street parking, a set of different scenarios with defined vehicle flows have been carried out.

In this sense, it has been determined a variable vehicle circulation flow between 500 and 1000 vehicles per lane. These values were defined in order to obtain a real recurrent level of vehicle flow in urban environments according to the Highway Capacity Manual.

Additionally, an important variable to remark is the routing of the vehicles that drive along the scenario considered. It has been defined that the $80 \%$ of the vehicles drive straight (no turns were made in the scenario) were the other $20 \%$ turned once ( $10 \%$ turned right and $10 \%$ turned left). Also, it is worth to say that the model has considered dynamic flow and the distribution of the flow is completely random.

\subsection{Definition of internal conditions}

Once the vehicle circulation as well as the routing has been fixed, the next step has been to define the illegal parking level existing in the chosen scenario and its location.

First of all, referred to the illegal parking level considered, the model has been run with different scenarios varying the level of illegality. This level has been determined according to real values (Suriñach et al.,2015 and Mesa Movilidad Madrid, 1997) between 0 and 1 vehicles/100 meters of road of the whole scenario; for the specific case of 1 vehicle per each 100 meters of the scenario, has to be taken into account that this implies an average of this parameter, the cars and spots when illegal parking is detected will be moving around during simulation.

As occurred with the flow circulation, the illegality on parking is also completely random distributed.

Referred to the location of illegality, this has been located in different spots of the evaluated model:

- Uniformly distributed in the whole scenario

- Concentrated in two roads

- Concentrated in one road

- Concentrated in a stretch of a road

It is worth to remark that independently of the studied case location of the illegality, the level of illegality in all cases has been defined as the illegality related to the whole scenario and not as the one referred just to the roads where the illegality appears.

Finally, referred to the sections where the travel time is evaluated, the location of these sections considered are:

- Whole scenario

- Illegal location

- > 200 meters to illegal location

\subsection{Scenario running and simulation}

Once defined and modeled, the different scenarios have been run. For each of them a total amount of 5 simulations have been carried out (each simulation has random vehicle 
distribution entries as well as location, duration and timeline of the illegal vehicles registered.

During the scenario simulation, the driving time data referred to 12 different stretches has been obtained. As it will be presented during the paper, this data will be used to evaluate the affectation of illegal parking in different levels.

\section{Calculation}

The parameters evaluated are the following:

- Affectation of the illegal parking depending on the vehicle circulation flow: a specific scenario of location of illegality has been fixed - concentrated in one road -. In this scenario, vehicle flow and level of illegality have been evaluated together in order to determine the crossing effects

For this evaluation only an illegal location has been considered but different vehicle flow values have been studied, however different levels of illegality have been studied

- Affectation of the illegal parking depending on the illegal parking location: for a determined vehicle flow (the amount considered has been 750 vehicles per lane), the crossing effects of level of illegal parking and the location of the illegality committed have been taken into account

In this evaluation, the vehicle flow has been fixed but several illegal parking locations have been studied. Different levels of illegality have been considered

\section{Results and discussion}

\subsection{Affectation of the illegal parking depending on the vehicle circulation flow}

In this first evaluation, the common characteristic of the scenario studied is that illegally parking is concentrated in one road (see figure below). Having this in mind, for different vehicle flow cases, traveling time of several road stretches have been evaluated in order to obtain the effect of illegal parking depending on the location of these stretches. 


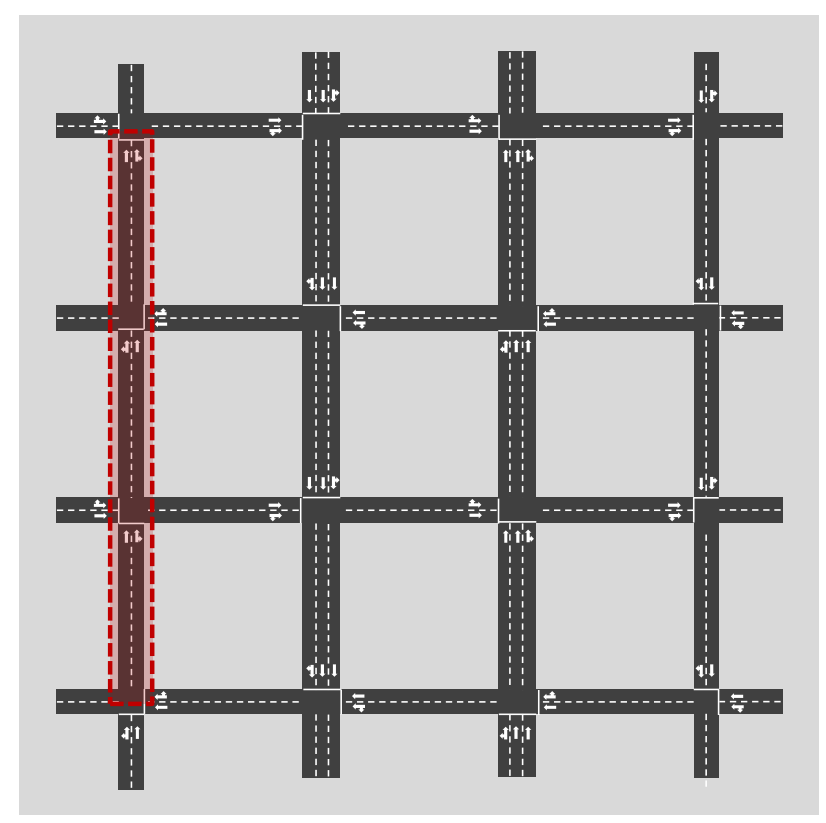

Figure 2. Location of illegal parked vehicles

According to the description made, the effect of illegal parking in travel time has been assessed for different flows as well as different sections. 

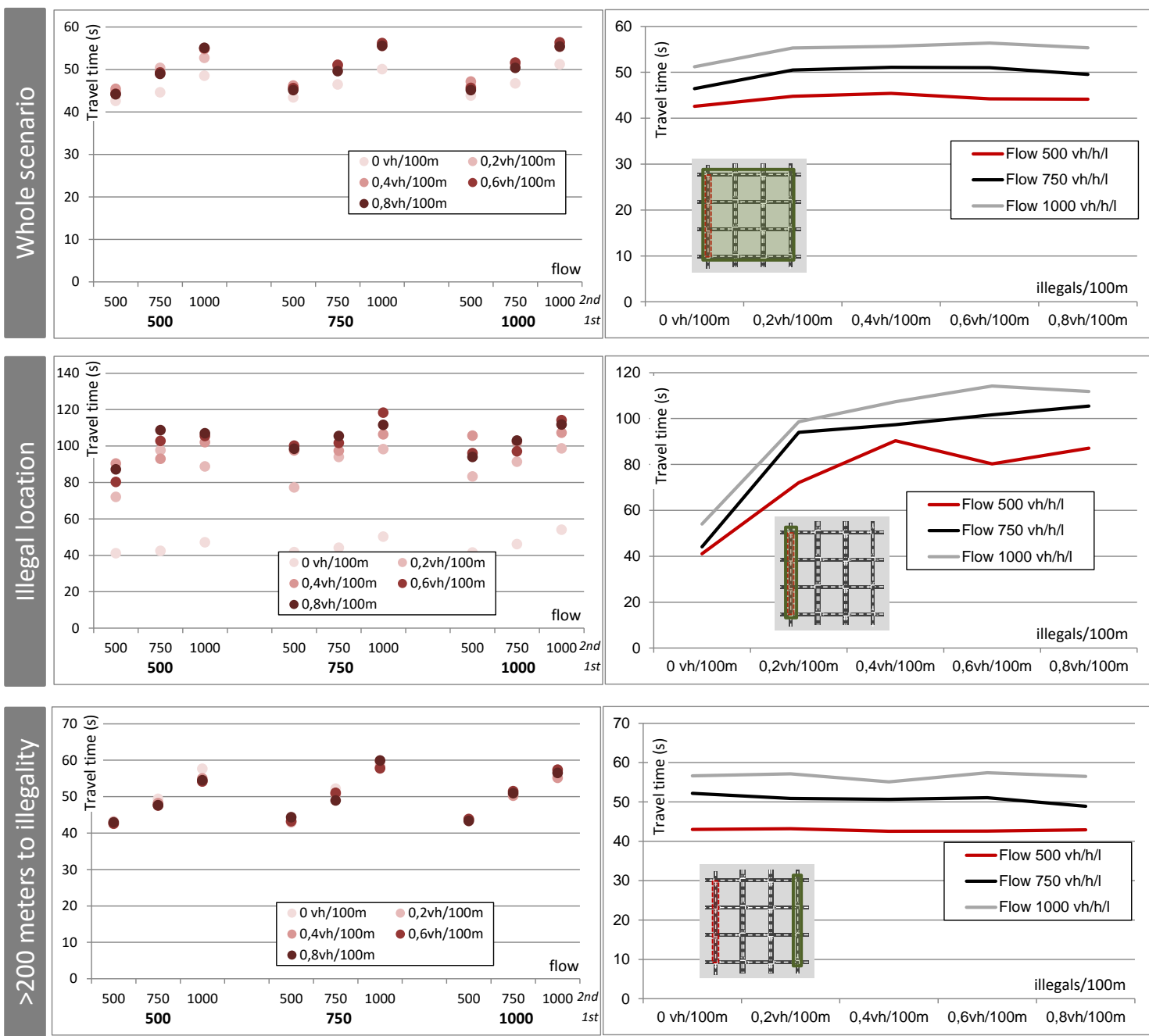

Figure 3. For different section case location, travel time against flow circulation for different illegal parking levels (left) and travel time against illegality level for fixed flow values (right)

Based on the detection of a certain level of illegality in a concrete road of the scenario (see figure above), the evaluation of the effect of illegal parked vehicles depending on the flow and the section studied shows the following elements:

- The effect of illegality in the different scenarios shows an invariable pattern for which a drastic increase of travel time is detected when the first illegality appears and an stabilization of the effect beyond this point

- It is worth to remark that the illegal parking effect on different circulation flows, appears to be is the same pattern independently of the flow considered

This pattern is valid for the different stretches were travel time have been evaluated in this scenario

- As predictable, the key parameter in this case is the distance between the vehicles and the focus of illegal parking. 
The appearance of an illegal parking corresponding to 0.2 vehicles for each 100 road meters is equal to a flow circulation increase of 20-30\% depending on the real flow of the case studied. From this point beyond, the effect of increasing the illegal parking is equal to a growth less than $5 \%$.

It is worth to remark that the effect referred to illegal parking is greater with low circulation flows and lesser with bigger flows due to in this case the saturation point is closer.

\subsection{Affectation of the illegal parking depending on location of it for a uniform circulation flow}

For each scenario, it has been evaluated the effect of illegal parking depending on the location of it.

It is worth to remark that the ratio of illegal parking per length has been constant for the whole scenario independent of where this illegality has been located.

In this sense, the different cases of illegal parking location have been determined as it is showed in the figure below.

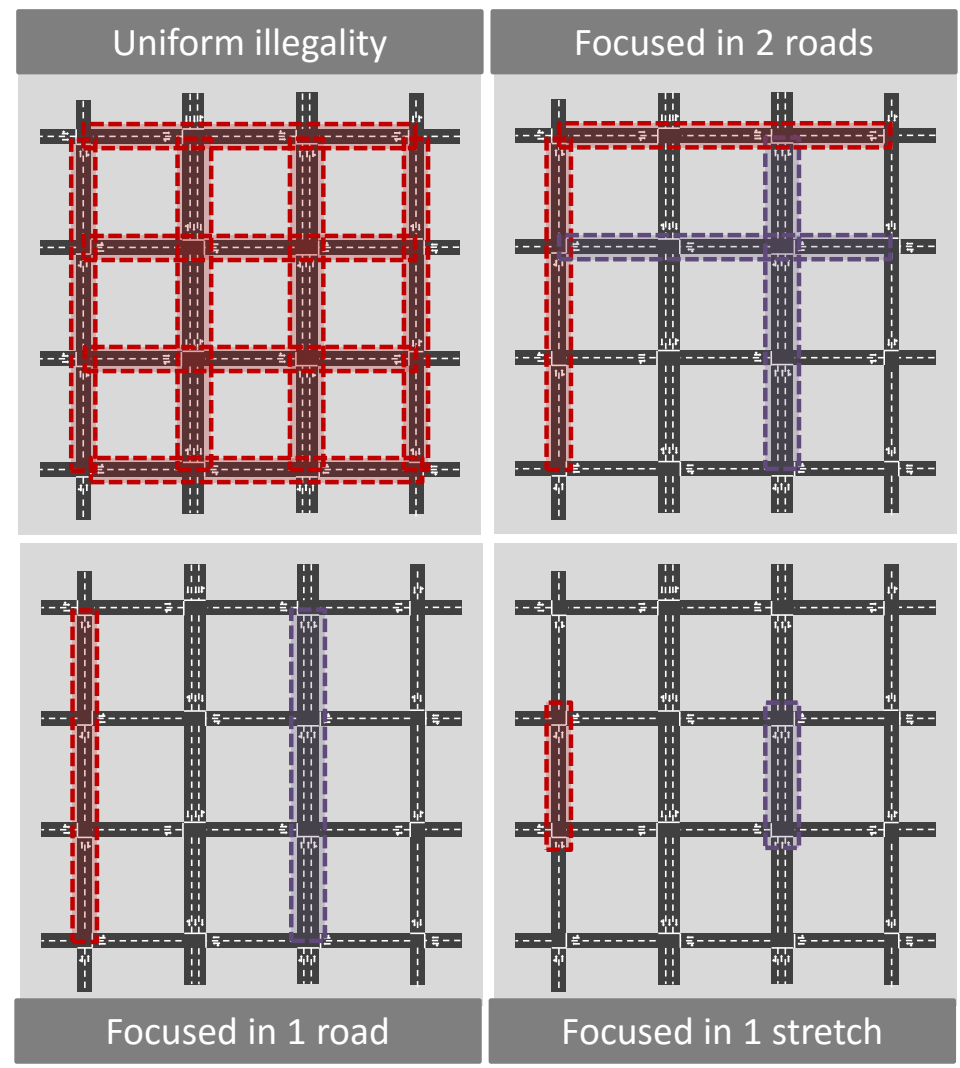

Figure 4. Location of illegal parked vehicles

For each of the scenarios considered above, the effect of increment of travel time has been evaluated each section case. 
In these scenarios, the different simulations have been carried out with the same circulation flow (750 vehicles per hour and per lane).

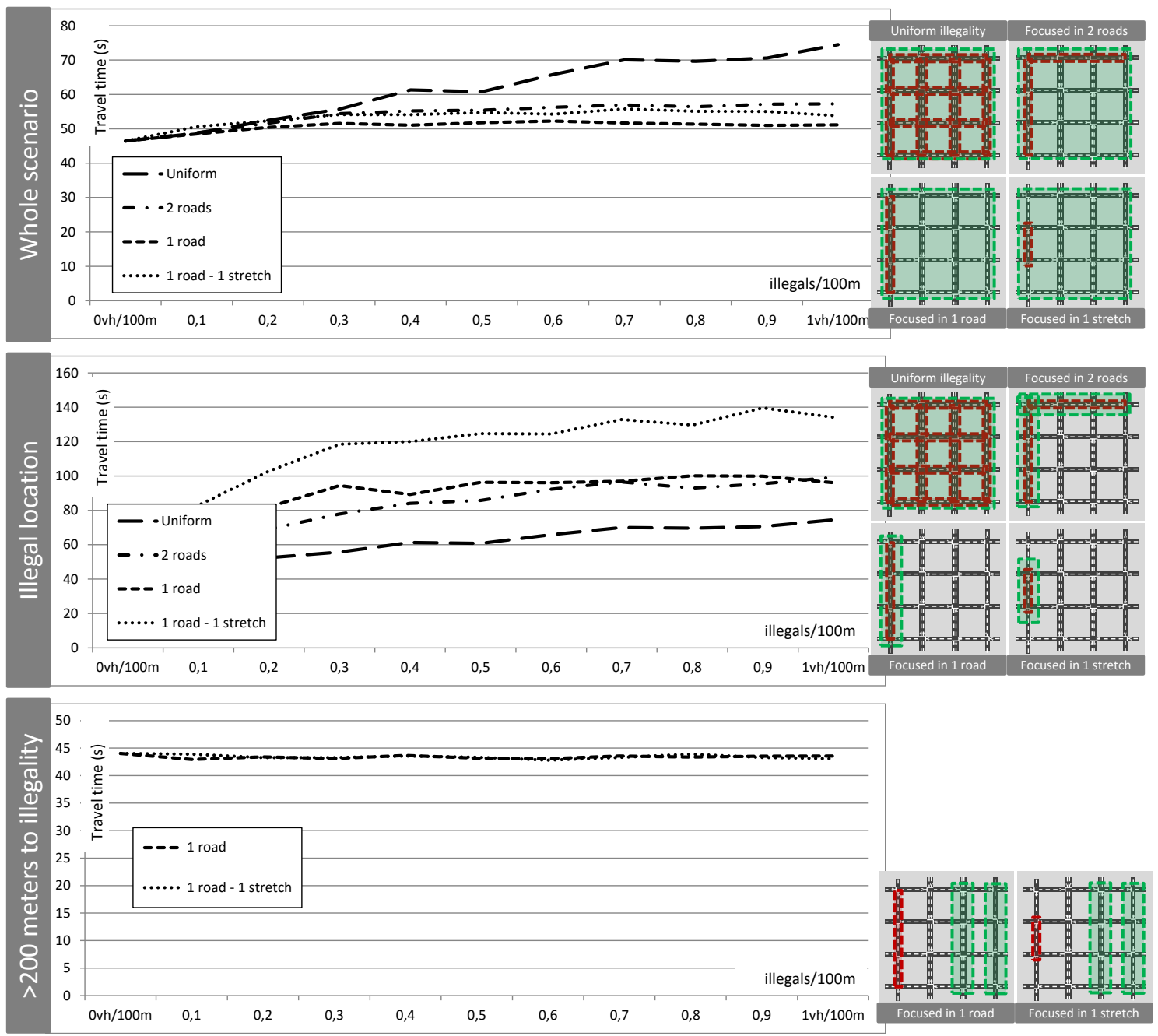

Figure 5. Travel time effect on vehicles depending on illegal parking level for different section cases

Regarding the results it is important to mention:

- Referred to the effect to the whole scenario, it is been detected that as long as the illegal parking increases, in all the different cases considered the illegality increases but in different manner. In the scenario where the illegal parked vehicles are distributed all along the scenario, the travel time increases the most when the illegality increases, in fact, it is the only scenario where the illegality keeps on affecting travel time as the illegal parking level goes up In the other hand, when the illegal parking is more concentrated in a smaller stretch, the effect on the whole scenario is lower

- As obvious, when the effect is only considered in the sections where illegality appears, the scenario with higher travel time increases is the one with illegal parked vehicles concentrated in the smallest stretch 
- When the evaluation is made to the stretches not affected directly by illegal parking (distance more than 200 meters), it has not been detected any effect referred to illegality in this stretches

From this data, it has been found that the effect on travel time in the vehicles is superior when an illegal parking location is uniformed distributed in the whole scenario.

The increase on travel time is a 50\% higher on the scenario of 1 vehicle per each 100 meters than the no illegality scenario. Referred to the difference between different illegality locations, it is worthy to remark that the values could differ about a $25 \%$.

Finally, it is important to say, based on the effect that illegality has in the different stretches, that the effect of illegality is detected only next to the point where appears and the indirect implications are null.
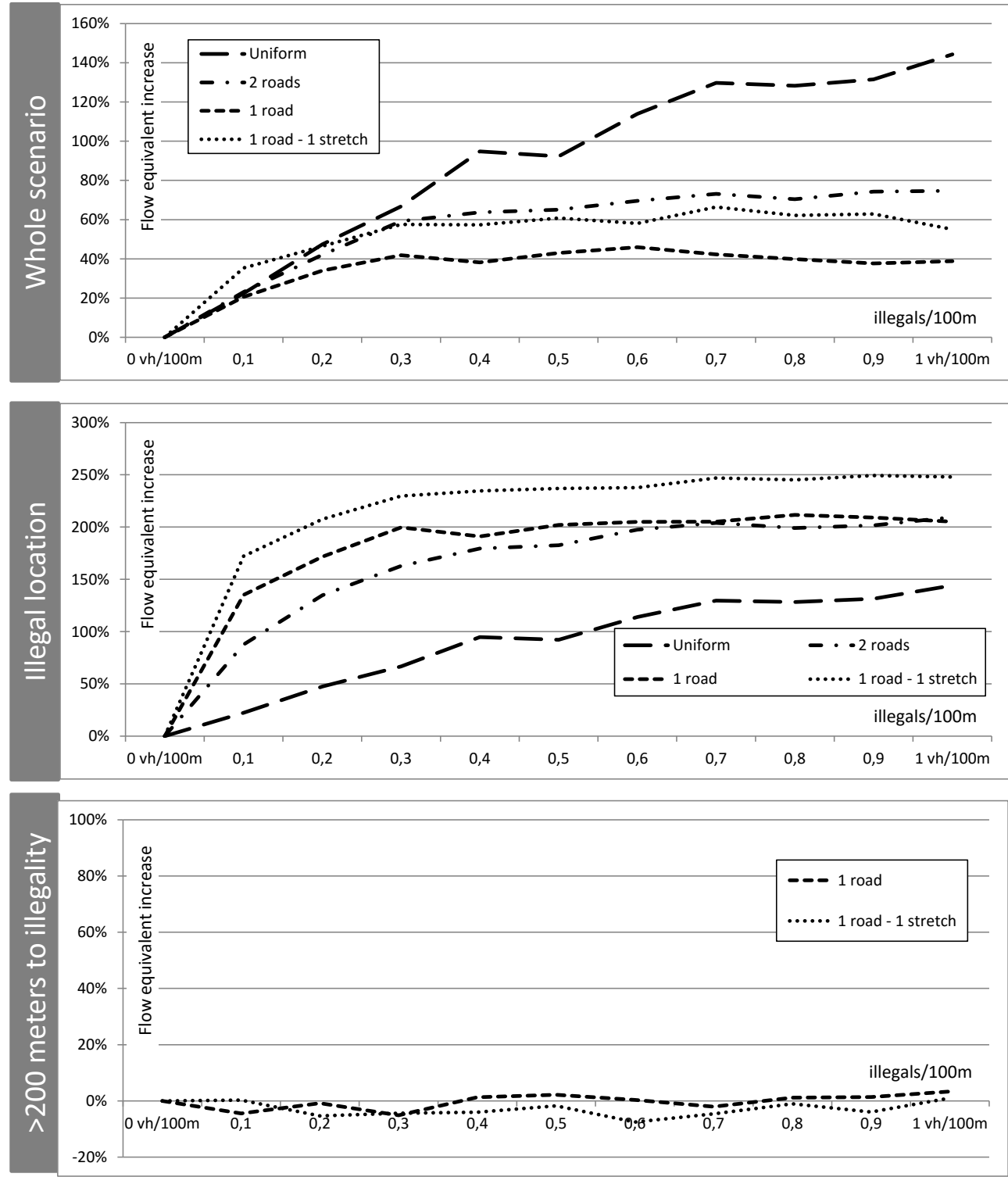

Figure 6. Relation between illegality level and flow-equivalent increase 
In order to obtain the effect on travel time of illegal parking compared to the effect of flow circulation increasing, a relation between flow increasing and illegal parking effect has been obtained. The main result of this is to transform the effect of illegal parking in flow growth in order to know the additional flow that a road could accept with no travel time modification if its illegal parking vehicles are erased.

In this sense, for the whole scenario, the appearance of illegal parking implies an increase of the equivalent-flow that varies depending on the case considered. For the uniformly distributed illegal parking case, the one with a worst effect in the urban environment evaluated, the flow-equivalent effect is equal to increase the real flow more than a $120 \%$ for an illegal parking value of 1 vehicle per 100 meters. The effect of illegal parking is constant as it increases ( $14 \%$ of flow per 0,1 vehicles/ 100 meters).

It is worth to remark that a case with the illegal parking more concentrated in specific stretches implies that the effect is lower and stabilized when the illegal parking achieves a fixed value (from 0,2 to 0,3 vehicles per 100 meters).

\section{Conclusions}

The strategy of illegal parking location with the lowest effect to the vehicles that circulate for the roads is the one related to locate the indiscipline in concentrated stretches not located in the main roads. In this case, it is worthy to remark that for a certain levels of illegality parking $(0,2-0,3 \mathrm{vh} / 100 \mathrm{~m})$, the difference between the several strategies evaluated is non-existing.

Based on the obtained results for the studied scenario, when the illegality has values around 0,2-0,3 vehicles per each 100 meters, the strategy of concentrated illegality reduces its negative effect (expressed as an increasing of circulation flow with a scenario without illegality) from 9 to $11 \%$ for each 0,1 vehicles per each additional 100 meters.

The effect on travel time depending on the illegal parking level has a specific behavior for each location case; in the cases where the illegality is concentrated is for low levels of illegal parking when this has a strong affectation on travel times. When the illegality level increases, in these cases, the additional effect is lower. The illegality affects strongly next to where appears but has almost no effect in the travel time.

In order to mitigate the effect that the illegal parking has in the travel time of vehicles, a double action is suggested depending on the illegal parking level existing in the zone.

If the illegal parking level is more than 0,25 vehicles per each 100 meters, the best option to reduce the effect on circulation travel times is to concentrate the illegal vehicles in a reduced spaces .

If the illegal parking level is less than 0,25 vehicles per each 100 meters, no strategy policies related to illegal parking location can be successfully made; in this sense, the only option to reduce the effect is to set up different mitigation policies in order to directly reduce the value of illegal parking level. 


\section{Future investigation}

As a future investigation lines derived of the present article, the following ideas are proposed:

- Develop a methodology to be able to implement the best strategy depending on the urban scheme in order to reduce the effect of illegal parking

- To make an analysis of a scenario in a real urban scheme in order to evaluate the effect of illegal parking as well as how it would affect an optimal indiscipline distribution in the whole scenario 


\section{References}

Sener (2010) Actualization of social and environmental transport costs in Barcelona Metropolitan Zone. $A M B$.

European Metropolitan Transport Authorities. EMTA Barometer. N 7, Paris, 2012

IERMB. Working day mobility survey. EMEF 2011, nº 9. Barcelona, 2012.

Mesa Movilidad Madrid (2007). Reduction of illegal on-street parking. Madrid.

Prevention, Security and Mobility Department (2002). Basic urban network in Barcelona. Barcelona.

Transport Research Board (2010). Highway Capacity Manual. The National Academies of Sciences, Engineering and Medicine. Washington.

Suriñach, J., Alcañiz, M., Guillén, M. (2015) Illegal parking evolution in Barcelona $\mathrm{n}^{\circ}$ 72. Spanish Econometry, Statistics and Economy Department. University of Barcelona. Barcelona

A.Albiol et al. (2015). Detection of Parked Vehicles using spatio-temporal maps. Valencia

J. Wang, F. Chen, (2012). Multi-Vehicle Tracking Based Traffic Illegal Parking Detection, Advanced Materials Research, Vols. 532-533, pp. 540-545

D. Shoup (2005). The High Cost of Free Parking. Chicago

Willeke, R. (1984) Soziale Kosten und Nutzen der Siedlungsballung und des Ballungsverkehrs. Frankfurt. 